\title{
Contribution of riboflavin supply pathways to Vibrio cholerae in different environments
}

\author{
Andrés Fuentes Flores ${ }^{1}$, Ignacio Sepúlveda Cisternas ${ }^{1,2}$, José Ignacio Vásquez Solis de Ovando ${ }^{1}$, Alexia Torres ${ }^{1}$ \\ and Víctor Antonio García-Angulo ${ }^{1,3^{*}}$
}

\begin{abstract}
Background: The waterborne diarrheagenic bacterium Vibrio cholerae, cause of the pandemic cholera disease, thrives in a variety of environments ranging from estuarine waters to the human intestinal tract. This species has two ways to obtain the essential micronutrient riboflavin, de novo biosynthesis and environmental uptake through the RibN importer. The way these functions interrelate to fulfill riboflavin needs in different conditions in this species is unknown.

Results: This study analyzed the contributions of riboflavin biosynthesis and transport to the culturability of Vibrio cholerae in river and seawater in vitro and in the Caenorhabditis elegans nematode host model. Elimination of the ribD riboflavin biosynthetic gene renders the bacteria riboflavin-auxotrophic, while a ribN mutant strain has no growth defect in minimal media. When growing in river water, deletion of ribD causes an impairment in culturability. In this condition, the $\triangle$ ribN strain has a defect to compete against a wild type strain but outcompetes the $\triangle r i b D$ strain. The latter effect is inverted by the addition of riboflavin to the water. In contrast, growth in seawater causes a loss in culturability independent of riboflavin biosynthesis or transport. In the C. elegans model, only the $\triangle r i b D$ strain is attenuated.
\end{abstract}

Conclusion: Results indicate that while riboflavin biosynthesis seems to outweigh riboflavin uptake, the latter may still provide a selective advantage to $V$. cholerae in some environments.

Keywords: Riboflavin transport, Biosynthesis, Vibrio cholerae, Vitamin B2, Environmental fitness

\section{Introduction}

Riboflavin, also named vitamin B2, is an essential micronutrient. This vitamin serves as precursor for biological electron transfer cofactors, mainly flavin mononucleotide (FMN) and flavin adenine dinucleotide (FAD). These products are required for several redox enzymatic reactions. The myriad of cellular cues that require flavins include carbon metabolism, oxidative stress protection, photosensitization and metabolism of other vitamins $[1,2]$. To accomplish its riboflavin demands, many bacteria may use the riboflavin biosynthetic pathway (RBP)

\footnotetext{
*Correspondence: victorgarcia@med.uchile.cl

${ }^{3}$ Facultad de Medicina Norte, Pabellón L. Santiago de Chile, ZIP 8380453 Santiago, Chile

Full list of author information is available at the end of the article
}

comprised by proteins encoded by the ribDEABH genes, while others lack the RBP but instead encode riboflavin importer proteins [3-10]. Frequently riboflavin biosynthesis and uptake abilities coexist in bacteria, but the way these two biological functions coordinate to provide riboflavin has been scarcely studied [6, 11-13]. Thus, while either riboflavin biosynthesis or uptake can be used to support life in many bacterial species, their relative importance in the so called riboflavin-opportunistic species [14] has not been established. It is conceivable that riboflavin uptake may help saving metabolic energy in a riboflavin prototrophic species and also it may provide riboflavin for specific cellular functions $[5,6,12,14,15]$.

Vibrio cholerae is a pandemic pathogen causing cholera, an acute diarrheal disease responsible for up to 
120,000 deaths every year worldwide [16]. This bacterium survives in fresh water and marine environments where it may form biofilms in biotic and abiotic surfaces and also grow in planktonic form [17-19]. After human consumption, $V$. cholerae colonizes and replicates in the host intestine where it expresses virulence factors, most notorious the cholera toxin and the toxin corregulated pilus, responsible for diarrhea and bacterial initial intestinal adhesion [20]. Given the wide spectrum of conditions that faces during its life cycle, $V$. cholerae likely must deal with variable nutrient availability. This organism is a riboflavin-prototrophic bacterium encoding a full RBP which features two putative $r i b B$ and $r i b A$ genes. In addition, it is also able to internalize riboflavin using the RibN importer [7, 21, 22]. In this species, the complexity of the RBP and its co-occurence with the riboflavin uptake ability may be related to the intrinsic variations of riboflavin concentrations featured by the wide spectrum of environments inhabitable to the pathogen. Thus, $V$. cholerae comprises an interesting case of study for the overlap of riboflavin provision pathways in bacteria. Recently, we reported that the presence of extracellular riboflavin downregulates the monocistronic gene $r i b B$, while having no effect on the expression of the RBP genes in the main riboflavin biosynthetic operon nor on ribN [22]. Nonetheless, no study has assessed the role of the riboflavin provision pathways of $V$. cholerae in different environments. In this report, we started defining the individual contributions of riboflavin biosynthesis and of the RibN riboflavin importer to $V$. cholerae by assessing the fitness of $\triangle r i b N$ and $\triangle r i b D$ strains in a set of different conditions, namely seawater and river water microcosms and the Caenorhabditis elegans host model.

\section{Methods}

Bacterial strains, plasmid construction and growth curves This work used WT $V$. cholerae $\mathrm{N} 16961$ and its $\Delta r i b N$ and $\Delta r i b N:: k a n$ derivative strains [22]. $V$. cholerae $\triangle r i b D$ was constructed by homologous recombination using the same protocol as that for ribN mutants [22, 23] and primers ribDH1P1 (5'- ATGCCTATGTTTACCTCTTTTGATCATCAAATGATGTCTCGCGCGGTGTAGGCTGGAGCTGCTTC-3') and ribDH2P2 (5' - C TAATC T T T TG T T T T TGG GG TGGCGAT GATCCGCAAATCTGCCCCCATATGAATATCCTCCTTAG-3'). The eliminantion of the ribD gene was corroborated by PCR using primers nrdR Fw and ribE Rv [22] flanking the deletion. To construct plasmid pB1ribD, ribD from $V$. cholerae N16961 was amplified by PCR from genomic DNA and primers nrdR Fw and ribE Rv. The resulting PCR fragment was cloned into pGEM T Easy (Promega) according to manufacturer's instructions to generate pGribD. Next, the fragment containing ribD was excised from this plasmid by ApaI and SpeI digestion and subcloned into the same sites of pBBR1 MCS 1 . This construction was corroborated by sequencing. For growth curves in $\mathrm{T}$ minimal media [24], the indicated bacterial strains were grown overnight on LB plates at $37^{\circ} \mathrm{C}$. Then, they were inoculated in $5 \mathrm{ml}$ fresh LB broth and incubated at $37{ }^{\circ} \mathrm{C}$ at $160 \mathrm{rpm}$ until they reached an optical density (O.D.) of $\sim 0.7$. Next, cultures were centrifuged at 12,000 rpm, supernatant discarded and pellet washed twice with $\mathrm{T}$ media and resuspended in fresh $\mathrm{T}$ media. $100 \mu \mathrm{l}$ of these cultures were used to inoculate $50 \mathrm{ml}$ of $\mathrm{T}$ media or $\mathrm{T}$ media with different riboflavin concentrations $(0,0.01,0.1$, or $2 \mu \mathrm{M})$ as indicated in $125 \mathrm{ml}$ flasks, incubated at $37{ }^{\circ} \mathrm{C}$ on an orbital shaker at $160 \mathrm{rpm}$ and O.D. determined at the indicated time points.

\section{Culturability on seawater and river water assays}

The indicated strains were grown overnight in LB plates. Next, a colony was inoculated in $5 \mathrm{ml}$ of LB broth and growth at $37{ }^{\circ} \mathrm{C}$ at $160 \mathrm{rpm}$ until a D.O. 0.7. These cultures were washed twice and resuspended in sterile water. $50 \mu \mathrm{l}$ were used to inoculate $50 \mathrm{ml}$ of filter-sterilized water from Mapocho river or Valparaiso bay, Chile, in Erlenmeyer flasks. Flasks were incubated at $25^{\circ} \mathrm{C}$ with soft shaking $(50 \mathrm{rpm})$. Initial inocula were determined by serial dilution platting on LB plates and incubation at $37^{\circ} \mathrm{C}$ for $18 \mathrm{~h}$ and culturability of the strains was assessed in the same way at the indicated time points. Importantly, $V$. cholerae is able to enter viable but non-culturable (VBNC) state $[25,26]$. In this state, the metabolic activity is reduced in order to survive harsh environments and bacteria do not grow in subsequent transfers to rich media [26]. Thus, we thoroughly use the term "culturability" in reference to UFC counts, as this term includes metabolically active bacteria and excludes both VBNC and dead cells. For competition experiments, $25 \mu \mathrm{l}$ of each strain obtained as those for single inoculations were added to the same flask. In such experiments, the $\Delta r i b N:: k a n$ strain was used [22]. For initial inocula and also for each time point, serial dilutions up to $10^{-6}$ were plated in order to obtain isolated colonies. For each determination, 200 isolated colonies were replicated in $\mathrm{T}$ medium to determine the fraction of each strain in the culture. Colonies of the $\triangle r i b N$ and $\triangle r i b D$ strains were identified by testing for kanamycin resistance and riboflavin auxotrophy, respectively.

\section{Real time PCR}

Vibrio cholerae WT was grown in $50 \mathrm{ml}$ of $\mathrm{T}$ media at $37^{\circ} \mathrm{C}$ and $160 \mathrm{rpm}$ until D.O. $=0.7$ and on 3 beakers with $50 \mathrm{ml}$ Mapocho river water each until day 3 as described previously. $1 \mathrm{ml}$ of $\mathrm{T}$ and $150 \mathrm{ml}$ of river water were centrifuged, washed twice, RNA extracted with the Thermo 
Scientific Genejet RNA purification kit and digested with Turbo DNA-free DNAase according to manufacturer's instructions. Next, complementary DNA was synthesized using AffinityScript QPCR cDNA Synthesis kit from Agilent Technologies. RT-PCR was performed using the Brilliant II SYBR Green QPCR Master Mix kit in a One-Step Applen Biosystems (Life Technologies) thermocycler. A reaction without reverse transcriptase was included for each sample in each run as a control. Relative expression in the indicated conditions was determined using the $\Delta \Delta \mathrm{Ct}$ method as developed before [27]. Measurements were normalized using the 16s rRNA gene. The sets of primers used for RT-PCR were ribN Fw/ribN Rv for ribN, ribD Fw/ribD Rv for ribD and 16s Fw/16s Rv for 16s [22].

\section{C. elegans survival assays}

$6 \mathrm{~cm}$ Petri dishes with NGM medium [28] containing $100 \mu \mathrm{l}$ of overnight cultures of $E$. coli OP50 or the indicated $V$. cholerae strains were incubated for $18 \mathrm{~h}$ at room temperature. Next, 30-32 L4 larvae of C. elegans strain $\mathrm{N} 2$ were added to each plate and incubated at $25^{\circ} \mathrm{C}$. The viability of the worms was scored at the indicated times by response to touch. Animals were transferred to fresh plates every $72 \mathrm{~h}$ to avoid progeny interference.

\section{Results}

To start defining the individual contributions of riboflavin biosynthesis and of the RibN riboflavin importer to the fitness of $V$. cholerae, we evaluated the effect of ribN and ribD deletions in different conditions. First, the riboflavin requirements of these strains were evaluated on defined T minimal medium [24] (Fig. 1). Elimination of ribN produced no growth defects compared to the WT strain. However, disruption of riboflavin biosynthesis by the elimination of ribD prevented V. cholerae from growing in $\mathrm{T}$ medium. Addition of increasing amounts of riboflavin to the medium gradually restored the ability of the $\Delta r i b D$ strain to grow. Addition of 0.01 or $0.1 \mu \mathrm{M}$ riboflavin produced only slight increases in the growth capacity, while $2 \mu \mathrm{M}$ riboflavin allowed the $\Delta r i b D$ strain to grow to WT levels. This result reflex the ability of this strain to internalize riboflavin. Also, complementation of the $\triangle r i b D$ strain with the multicopy plasmid pB1ribD encoding ribD from $V$. cholerae restored growth in the absence of exogenous riboflavin, although with a lower rate compared to the WT. The lack of full complementation could indicate that the genetic charge generated by the multicopy plasmid may be disadvantageous to the bacteria. Besides, the overproduction of highly reactive riboflavin biosynthesis intermediaries due to overexpression of RibD could affect growth. Riboflavin biosynthesis intermediaries may be toxic to the cell [29].

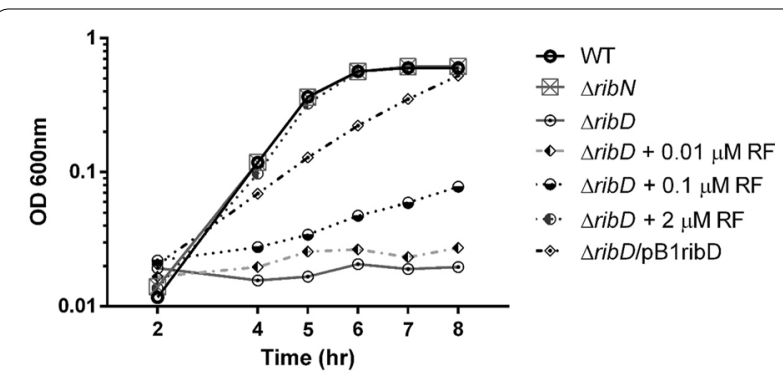

Fig. 1 Growth curves of N16961 V. cholerae WT and its derivative strains in minimal $\mathrm{T}$ medium. The strains were inoculated in T media or T media plus riboflavin (RF) as indicated and incubated at $37^{\circ} \mathrm{C}$ and O.D. measured hourly. The average of three independent experiments is shown

Next, we evaluated the fitness of these strains inoculated in river water. The WT strain showed an initial increase in colony forming units (CFU) numbers of approximately $2.5 \mathrm{log}$ after the first day of incubation (Fig. 2a). This culturability level was thereafter maintained around the same until day 10 . The $\Delta r i b N$ strain showed a similar pattern of culturability. However, the $\triangle r i b D$ displayed a gradual decrease in CFU numbers after 1 day of incubation. At day 10, CFU numbers were around 100-fold reduced for the $\triangle r i b D$ compared to WT. On one hand, addition of riboflavin to water did not increase culturability of the WT and $\Delta r i b N$ strains (Fig. 2b, c). On the other hand, the culturability defect displayed by the $\triangle r i b D$ was abrogated by addition of $2 \mu \mathrm{M}$ (Fig. 2c) or even $0.1 \mu \mathrm{M}$ riboflavin to the water (Fig. 2b). Following, we performed competition experiments where two strains were co-inoculated. Competition assays between a mutant strain and its WT parental are usually performed in order to identify defects to colonize a niche. Competition assays are more sensitive than single strain assays as direct competition against a fully capable strain exacerbates colonization impairments. Also, testing two strains within the same experimental unit usually decreases variation among time points and replicates [30]. In these co-culture experiments, the percentage of each strain remaining as culturable colonies at different time points was determined. In line with the result showing a defect to grow in plain river water, the $\Delta r i b D$ strain was gradually outcompeted by the WT strain from day 1 to day 10 (Fig. 2d, e). This outcome was delayed but not avoided by the addition of riboflavin to water. Meanwhile, the $\Delta r i b N$ showed a defect to compete against the WT strain manifested from day 1 and maintained the same until day 10 (Fig. 2f, g). This effect was independent of added riboflavin. When the two mutants were co-inoculated (Fig. $2 \mathrm{~h}, \mathrm{i}$ ), the $\Delta r i b D$ was progressively outcompeted by the $\Delta r i b N$. Nonetheless, addition 

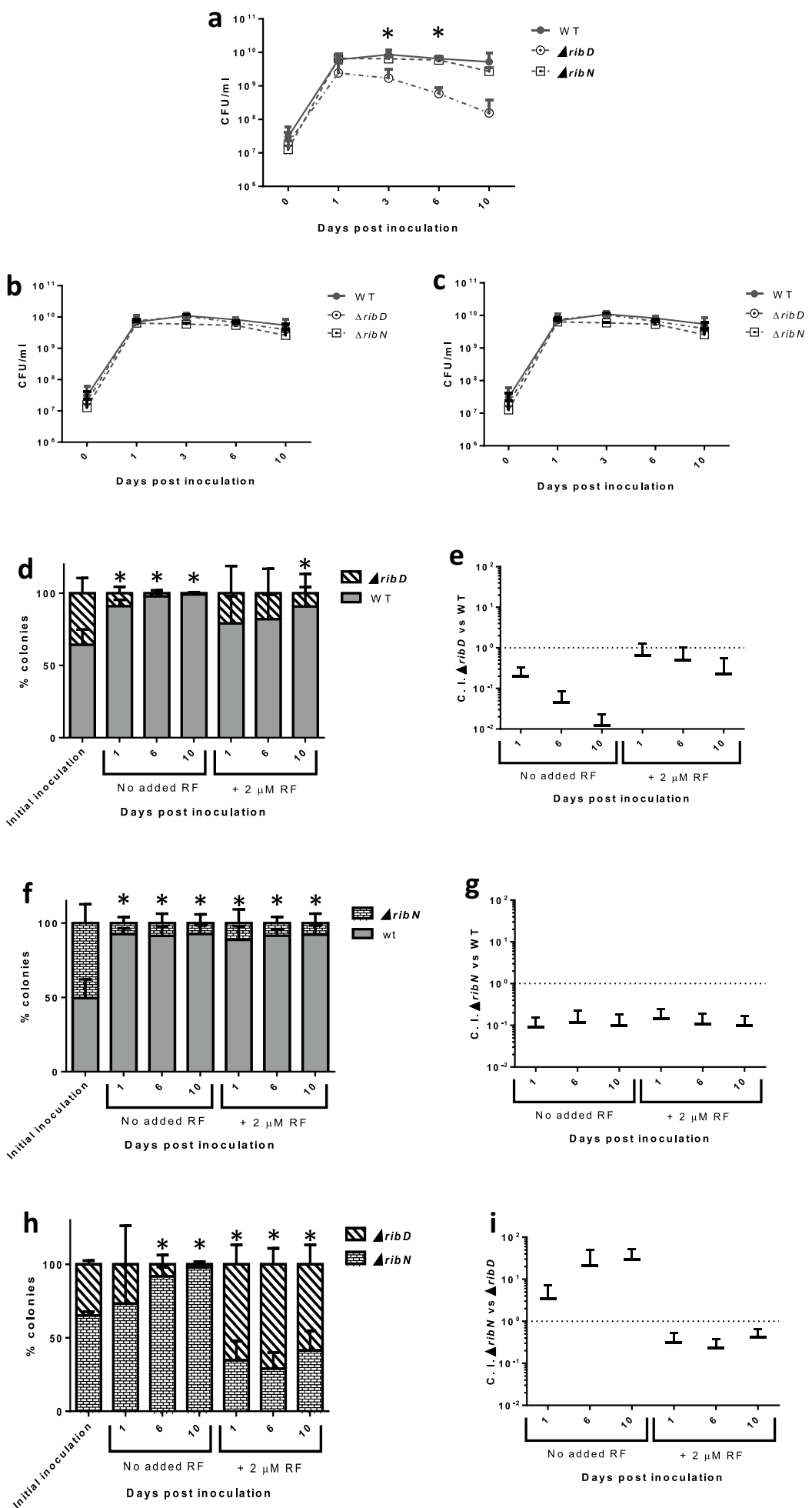

Fig. 2 Contribution of ribD and ribN to V. cholerae growth in river water. Culturability of $\mathrm{V}$. cholerae strains in filter-sterilized water from Mapocho river (a) and effect of $0.1 \mu \mathrm{M}(\mathbf{b})$ or $2 \mu \mathrm{M}$ (c) riboflavin (RF). Culturability of $\mathrm{V}$. cholerae in experiments of co-inoculation of strains in water from the Mapocho river with or without $2 \mu \mathrm{M}$ exogenous riboflavin and the same results expressed as competitive index (C.I.) for WT versus $\triangle$ ribD (d, e), WT versus $\triangle r i b N(\mathbf{f}, \mathbf{g})$ and $\triangle r i b D$ versus $\triangle r i b N(\mathbf{h}, \mathbf{i})$. The average and standard deviation of three independent experiments is shown. ${ }^{*}$ denotes statistically significant difference $(P<0.05)$ between WT and $\triangle r i b D(\mathbf{a})$ or between the indicated bar and the initial inoculum $(\mathbf{d}, \mathbf{f}, \mathbf{h})$ determined by t tests analysis 
of riboflavin overturned this pattern, with the $\Delta r i b D$ mantaining a small advantage to compete against the $\Delta r i b N$ strain. Yet, this leverage was lesser than the advantage displayed by the $\Delta r i b N$ over the $\Delta r i b D$ strain without exogenous riboflavin. We previously reported that extracellular riboflavin does not changes the expression of $r i b D$ nor of ribN [22]. In order to evaluate if river water induces changes in the expression of these riboflavin supply pathways, we determined the relative expression of both genes in $\mathrm{T}$ medium versus river water. Results showed a reduction in expression in river water. This reduction was similar for $r i b D$ and $r i b N$, with the expression in river water reduced to roughly one tenth of that in $\mathrm{T}$ medium (Additional file 1). To assess if growth in river water boosts the expression of any riboflavin provision pathway over the other, we also determined the ribD/ribN expression ratio in $\mathrm{T}$ medium and in river water. Results show that the ratio of $r i b D / r i b N$ expression in river water is similar to that in T medium (Additional file 2). Thus, river water seems to induce a general downregulation of riboflavin supply, while not inducing the overexpression of neither pathway.

When inoculated in seawater, the dramatic increase in culturability after 1 day of incubation observed in river water was not presented (Fig. 3a). Moreover, CFU were reduced at days 6 and 10 . This pattern of culturability was similar for all three strains irrespective of the presence of exogenous riboflavin (Fig. 3a, b). In competition experiments, both mutants were recovered at the same levels as the WT (Fig. 3c-f) and also when competing against each other independently of the addition of riboflavin (Fig. 3g, h).

Finally, we assessed the effect of the deletions on the lifespan of the C. elegans nematode. Feeding C. elegans with $V$. cholerae produces a moderate reduction in lifespan compared to the growth on E. coli OP50, the bacterial strain routinely used to feed $C$. elegans in the laboratory, i.e. the average time to reach $90 \%$ mortality was close to 14 days for $E$. coli but around 10 days for WT V. cholerae (Fig. 4). C. elegans growing on the $\Delta$ ribD strain had an increased lifespan, similar to when growing on E. coli, while the worms growing on $\Delta r i b N$ had a similar lifespan to growth on WT $V$. cholerae.

\section{Discussion}

The role of riboflavin transport in riboflavin prototrophic bacteria has started to be settled. In the riboflavin prototroph Corynebacterium glutamicum, the RibM riboflavin importer is able to provide riboflavin in the absence of endogenous biosynthesis, although the riboflavin intracellular levels are lower than in the wild type strain [13]. In this work, when growing in minimal media, exogenous riboflavin seems to fully replace the RBP but only at relatively high riboflavin media concentration $(2 \mu \mathrm{M})$. This seems a rather large concentration for a transporter to achieve full provision of a metabolite in physiological conditions. This is probably due to low substrate affinity, co-transport requirements or other unidentified factor. This is hard to hypothesize because the transport mechanism for the RibN family has not been studied. Previous works have estimated the riboflavin concentration in open sea water in $30 \mathrm{pM}$ and estuarine waters in a range of 45-128 pM [31,32]. This is well below the $2 \mu \mathrm{M}$ required to provide full growth to the $\Delta r i b D$ strain in vitro. Thus, we hypothesize that $2 \mu \mathrm{M}$ riboflavin is a concentration not likely found in environments where $V$. cholerae naturally thrives. Results obtained in river water indicate that endogenous riboflavin biosynthesis is both required and sufficient to maintain culturability of $V$. cholerae. In this condition, the strain lacking biosynthesis showed normal levels of culturability by day 1 , but showed a decrease from day 3 onward. Prior to inoculation on river water, bacteria are grown in LB medium, which is plenty of riboflavin. In such conditions, it may be expected that bacterial cells of all strains contain high riboflavin intracellular levels. A possible explanation for the culturability pattern of the $\Delta r i b D$ strain is that these vitamin levels may be sufficient for initial cell replication, but as time passes by and cellular riboflavin pools dilute, the strain unable to synthesize riboflavin starts to loose fitness. Notably, this result is analogous to that obtained by a previous study. While assessing the ability of a RBP-deficient Brucella abortus strain to survive inside macrophages, equal or even improved survival levels compared to the WT were observed at early time points. Later, survival dropped dramatically due to riboflavin depletion [33]. Strikingly, in river water $0.1 \mu \mathrm{M}$ riboflavin was sufficient to restore culturability to the $\Delta r i b D$ strain while this concentration only provided minimal rescue in $\mathrm{T}$ medium. This suggests than in some conditions, riboflavin transport is more efficient in fulfilling riboflavin needs. The analysis of the relative expression or ribN and ribD ruled out the possibility of an increased expression of the transporter in river water. Thus, it is conceivable that intracellular flavin needs may be reduced and/ or that an alternative redox cofactor becomes available in river water. In these conditions, RibN confers an advantage to compete against the WT strain. This suggests that part of the riboflavin required by the bacteria may be provided by uptake. However, the fact that the strain lacking riboflavin biosynthesis was outcompeted by the strain deficient in uptake suggests that biosynthesis outweighs internalization in these conditions. In spite of this, the addition of riboflavin overturning this pattern reinforces the notion that when growing in riboflavin rich environments, transport may confer an advantage 

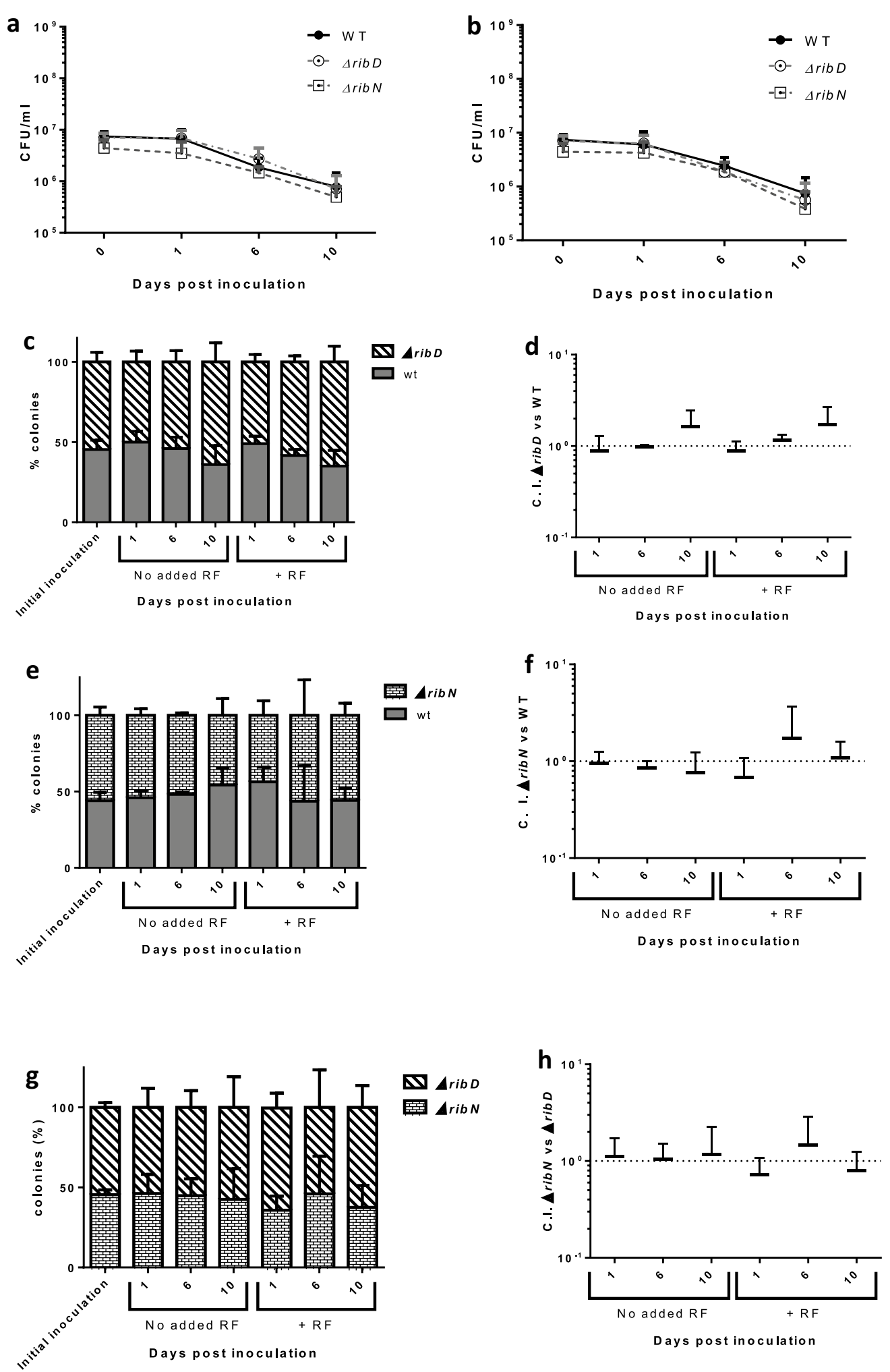

Fig. 3 Culturability of $V$. cholerae in seawater. Filter-sterilized seawater was incubated with the indicated strains for culturability and competition assays determinations. a Culturability of strains in seawater without exogenous riboflavin. $\mathbf{b}$ Culturability of strains in seawater plus $2 \mu \mathrm{M}$ riboflavin. c Culturability of strains in co-inoculation experiments of WT versus $\triangle r i b D, W T$ versus $\triangle r i b N$ and $\triangle r i b D$ versus $\triangle r i b N(\mathbf{c}, \mathbf{e}, \mathbf{g})$ and the same results expressed as C.I. (d, f, h) 


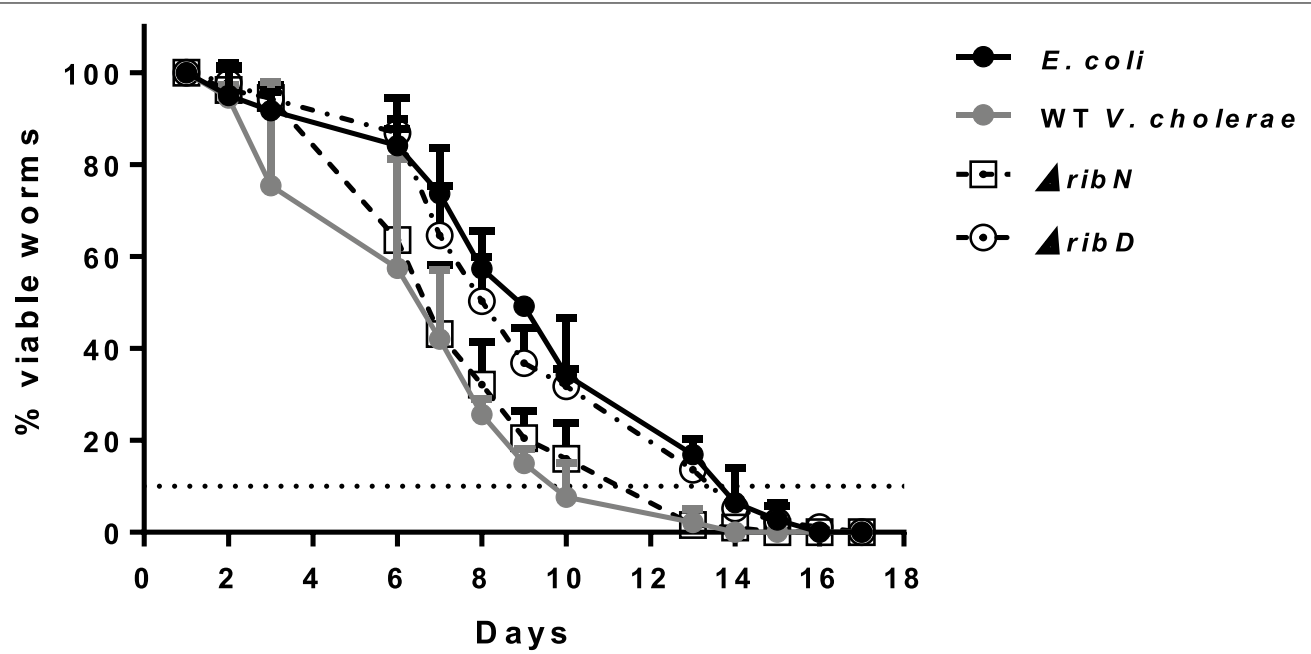

Fig. 4 Caenorhabditis elegans lifespan in E. coli and V. cholerae. C. elegans were fed bacterial lawns of the indicated strains and survival determined by response to touch recorded. Results shown are the average and standard deviation of six measurements

over biosynthesis. Notably, results obtained in similar experiments performed on seawater rendered a different outcome. Here, a clear reduction in culturability was observed for the three strains. Entrance of $V$. cholerae to VBNC state in filtered seawater has been reported before $[25,34]$. Thus, in our experiment, $V$. cholerae likely also rapidly enters the VBNC state. As both mutants lost culturability at the same rate as the WT in single inoculations, it seems that elimination of ribN or ribD does not affect this process. The fact that similar numbers of each strain are recovered during competition assays does not necessarily means that the ribD and ribN mutants are successfully competing against the WT strain, but this outcome is probably related to the fact that all strains are rapidly loosing culturability in this condition. In order to see fitness differences, strains likely need to be in conditions allowing active growth. Nonetheless, further experimental evidence is needed in order to better depict the role of riboflavin provision pathways in seawater.

Finally, results in the host model suggest that riboflavin biosynthesis is specifically required for lifespan reduction on C. elegans while lost of ribN does not attenuates the bacterial potential of lifespan reduction. Given its general impairment to grow in riboflavin-restrictive niches, the increased lifespan on the $\Delta r i b D$ strain may be due to a pleiotropic metabolic impairment rather than specific effects on virulence traits.

Overall, results indicate that riboflavin biosynthesis seems prevalent over riboflavin transport for fitness of $V$. cholerae in river water and on the C. elegans host model. Recently, a methicillin-resistant Staphylococcus aureus $\triangle$ ribD strain keeping an energy-coupling factorRibU riboflavin uptake system was shown to require a low concentration of exogenous riboflavin to achieve wild type growth levels. This strain was not impaired in a murine model of host colonization, indicating that in such biological system, riboflavin uptake may fully substitute for biosynthesis [35]. Thus, it may be expected that the relative relevance of the riboflavin supply pathways varies across bacteria and their specific niches. In $V$. cholerae, riboflavin transport provides an improvement to compete in river water. Moreover, when growing in riboflavin-rich conditions, using exclusively the RibN importer offers an advantage over solely biosynthesizing riboflavin and this may be better accomplished in natural environments. This result indeed suggests that an incentive for uptaking over biosynthesizing exists.

\section{Additional files}

Additional file 1. Expression of ribD and ribN is diminished in river water compared to expression in T minimal media. Expression of ribD and ribN in river water compared to expression in T media, assessed by RT-PCR as described in Methods. Media and standard deviation of three independent experiments are shown.

Additional file 2. River water does not changes the ribN/ribD expression ratio compared to growth in T minimal media. Relative expression of ribN compared to expression of ribD in T minimal media and river water as determined by RT-PCR. Media and standard deviation of three independent experiments are shown.

\section{Authors' contributions}

AFF performed experiments of culturability and competence in river water and seawater and C. elegans survival curves and discussed results. ISC performed RT-PCR experiments, discussed results, provided technical support and helped writing the paper. JIVSO performed minimal medium growth curves and discussed results. AT constructed the ribD mutant and discussed results. VAG conceived the study, analyzed results, constructed the pB1 ribD plasmid and wrote the manuscript. All authors read and approved the final manuscript. 


\begin{abstract}
Author details
${ }^{1}$ Programa de Microbiología y Micología, Instituto de Ciencias Biomédicas, Universidad de Chile, Santiago, Chile. ${ }^{2}$ Escuela de Biotecnología, Universidad Mayor, Campus Huechuraba, Santiago, Chile. ${ }^{3}$ Facultad de Medicina Norte, Pabellón L. Santiago de Chile, ZIP 8380453 Santiago, Chile.
\end{abstract}

\section{Acknowledgements}

We thank Dr. Andrea Calixto Mohor, from Universidad Mayor, Chile, for providing C. elegans N2 and E. coli OP50.

\section{Competing interests}

The authors declare that they have no competing interests.

\section{Availability of data and materials}

All data generated or analyzed during this study are included in this published article.

\section{Consent for publication}

Not applicable.

\section{Ethics approval and consent to participate}

Not applicable.

\section{Funding}

This study was funded by CONICYT FONDECYT Regular (Chile), Grant Number 1150818.

\section{Publisher's Note}

Springer Nature remains neutral with regard to jurisdictional claims in published maps and institutional affiliations.

Received: 8 September 2017 Accepted: 6 November 2017

Published online: 14 November 2017

\section{References}

1. Rodionov DA, Dubchak I, Arkin A, Alm E, Gelfand MS. Reconstruction of regulatory and metabolic pathways in metal-reducing $\delta$-proteobacteria. Genome Biol. 2004;5:R90.

2. Abbas CA, Sibirny AA. Genetic control of biosynthesis and transport of riboflavin and flavin nucleotides and construction of robust biotechnological producers. Microbiol Mol Biol Rev MMBR. 2011;75:321-60.

3. Burgess CM, Slotboom DJ, Geertsma ER, Duurkens RH, Poolman B, van Sinderen D. The riboflavin transporter RibU in Lactococcus lactis: molecular characterization of gene expression and the transport mechanism. J Bacteriol. 2006;188:2752-60.

4. Vogl C, Grill S, Schilling O, Stülke J, Mack M, Stolz J. Characterization of riboflavin (vitamin B2) transport proteins from Bacillus subtilis and Corynebacterium glutamicum. J Bacteriol. 2007;189:7367-75.

5. Jaehme M, Slotboom DJ. Diversity of membrane transport proteins for vitamins in bacteria and archaea. Biochim Biophys Acta. 2015:1850:565-76.

6. Gutiérrez-Preciado A, Torres AG, Merino E, Bonomi HR, Goldbaum FA, García-Angulo VA. Extensive identification of bacterial riboflavin transporters and their distribution across bacterial species. PLOS ONE. 2015;10:e0126124.

7. García Angulo VA, Bonomi HR, Posadas DM, Serer MI, Torres AG, Zorreguieta Á, et al. Identification and characterization of RibN, a novel family of riboflavin transporters from Rhizobium leguminosarum and other proteobacteria. J Bacteriol. 2013;195:4611-9.

8. Sun El, Leyn SA, Kazanov MD, Saier MH, Novichkov PS, Rodionov DA. Comparative genomics of metabolic capacities of regulons controlled by cis-regulatory RNA motifs in bacteria. BMC Genomics. 2013;14:597.

9. Rodionova IA, Li X, Plymale AE, Motamedchaboki K, Konopka AE, Romine MF, et al. Genomic distribution of B-vitamin auxotrophy and uptake transporters in environmental bacteria from the Chloroflexi phylum. Environ Microbiol Rep. 2015;7:204-10.
10. Deka RK, Brautigam CA, Biddy BA, Liu WZ, Norgard MV. Evidence for an $A B C$-type riboflavin transporter system in pathogenic spirochetes. MBio. 2013:4:e00615.

11. Vitreschak AG, Rodionov DA, Mironov AA, Gelfand MS. Regulation of riboflavin biosynthesis and transport genes in bacteria by transcriptional and translational attenuation. Nucleic Acids Res. 2002;30:3141-51.

12. García-Angulo VA. Overlapping riboflavin supply pathways in bacteria. Crit Rev Microbiol. 2017;43:196-209.

13. Takemoto $N$, Tanaka $Y$, Inui M, Yukawa $H$. The physiological role of riboflavin transporter and involvement of FMN-riboswitch in its gene expression in Corynebacterium glutamicum. Appl Microbiol Biotechnol. 2014;98:4159-68.

14. Anderson LN, Koech PK, Plymale AE, Landorf EV, Konopka A, Collart FR, et al. Live cell discovery of microbial vitamin transport and enzymecofactor interactions. ACS Chem Biol. 2016;11:345-54.

15. Hemberger S, Pedrolli DB, Stolz J, Vogl C, Lehmann M, Mack M. RibM from Streptomyces davawensis is a riboflavin/roseoflavin transporter and may be useful for the optimization of riboflavin production strains. BMC Biotechnol. 2011:11:119.

16. Ali M, Nelson AR, Lopez AL, Sack DA. Updated global burden of cholera in endemic countries. PLoS Negl Trop Dis. 2015;9:e0003832.

17. Teschler JK, Zamorano-Sánchez D, Utada AS, Warner CJA, Wong GCL, Linington RG, et al. Living in the matrix: assembly and control of Vibrio cholerae biofilms. Nat Rev Microbiol. 2015;13:255-68.

18. Chowdhury FR, Nur Z, Hassan N, von Seidlein L, Dunachie S. Pandemics, pathogenicity and changing molecular epidemiology of cholera in the era of global warming. Ann Clin Microbiol Antimicrob. 2017;16:10.

19. Silva AJ, Benitez JA. Vibrio cholerae biofilms and cholera pathogenesis PLoS Negl Trop Dis. 2016;10:e0004330

20. Clemens JD, Nair GB, Ahmed T, Qadri F, Holmgren J. Cholera. Lancet Lond Engl. 2017;390:1539-49.

21. Brutinel ED, Dean AM, Gralnick JA. Description of a riboflavin biosynthetic gene variant prevalent in the phylum Proteobacteria. J Bacteriol. 2013:195:5479-86.

22. Cisternas IS, Torres A, Flores AF, Angulo VAG. Differential regulation of riboflavin supply genes in Vibrio cholerae. Gut Pathog. 2017;9:10.

23. Datsenko KA, Wanner BL. One-step inactivation of chromosomal genes in Escherichia coli K-12 using PCR products. Proc Natl Acad Sci USA. 2000:97:6640-5.

24. WyckoffEE, Payne SM. The Vibrio cholerae VctPDGC system transports catechol siderophores and a siderophore-free iron ligand. Mol Microbiol. 2011;81:1446-58.

25. Xu H-S, Roberts N, Singleton FL, Attwell RW, Grimes DJ, Colwell RR. Survival and viability of nonculturable Escherichia coli and Vibrio cholerae in the estuarine and marine environment. Microb Ecol. 1982;8:313-23.

26. Wu B, Liang W, Kan B. Growth phase, oxygen, temperature, and starvation affect the development of viable but non-culturable state of Vibrio cholerae. Front Microbiol. 2016;7:404

27. Livak KJ, Schmittgen TD. Analysis of relative gene expression data using real-time quantitative $P C R$ and the $2-\triangle \triangle C T$ method. Methods San Diego Calif. 2001;25:402-8.

28. Brenner S. The genetics of Caenorhabditis elegans. Genetics. 1974;77:71-94.

29. Frelin O, Huang L, Hasnain G, Jeffryes JG, Ziemak MJ, Rocca JR, et al. A directed-overflow and damage-control $\mathrm{N}$-glycosidase in riboflavin biosynthesis. Biochem J. 2015;466:137-45.

30. Li Y-H, Tian X-L, Layton G, Norgaard C, Sisson G. Additive attenuation of virulence and cariogenic potential of Streptococcus mutans by simultaneous inactivation of the ComCDE quorum-sensing system and HK/RR1 1 two-component regulatory system. Microbiology. 2008;154:3256-65.

31. Sañudo-Wilhelmy SA, Cutter LS, Durazo R, Smail EA, Gómez-Consarnau L, Webb EA, et al. Multiple B-vitamin depletion in large areas of the coastal ocean. Proc Natl Acad Sci USA. 2012;109:14041-5.

32. Heal KR, Carlson LT, Devol AH, Armbrust EV, Moffett JW, Stahl DA, et al. Determination of four forms of vitamin B12 and other B vitamins in seawater by liquid chromatography/tandem mass spectrometry. Rapid Commun Mass Spectrom RCM. 2014;28:2398-404.

33. Bonomi HR, Marchesini MI, Klinke S, Ugalde JE, Zylberman V, Ugalde RA, et al. An atypical riboflavin pathway is essential for Brucella abortus virulence. PLOS ONE. 2010;5:e9435.

34. McCarthy SA. Effects of temperature and salinity on survival of toxigenic Vibrio cholerae O1 in seawater. Microb Ecol. 1996:31:167-75. 
35. Wang H, Mann PA, Xiao L, Gill C, Galgoci AM, Howe JA, et al. Dualtargeting small-molecule inhibitors of the Staphylococcus aureus FMN riboswitch disrupt riboflavin homeostasis in an infectious setting. Cell Chem Biol. 2017;24:576-88.

Submit your next manuscript to BioMed Central and we will help you at every step:

- We accept pre-submission inquiries

- Our selector tool helps you to find the most relevant journal

- We provide round the clock customer support

- Convenient online submission

- Thorough peer review

- Inclusion in PubMed and all major indexing services

- Maximum visibility for your research 